\title{
Effects of vacancies on phonon entropy of $\mathrm{B2} \mathrm{FeAl}$
}

\author{
M. S. Lucas and O. Delaire \\ Oak Ridge National Laboratory, 1 Bethel Valley Road, Oak Ridge, Tennessee 37831, USA
}

M. L. Winterrose, T. Swan-Wood, M. Kresch, I. Halevy, and B. Fultz

California Institute of Technology, W. M. Keck Laboratory, P.O. Box 138-78, Pasadena, California 91125, USA

Jingzhu $\mathrm{Hu}$

National Synchrotron Light Source, University of Chicago, Upton, New York 11973, USA

M. Lerche, M. Y. Hu, and M. Somayazulu

Geophysical Laboratory, Carnegie Institution for Science, Washington, D.C. 20015, USA

(Received 22 June 2009; revised manuscript received 17 November 2009; published 21 December 2009)

\begin{abstract}
The phonon density of states (DOS) and phonon entropy of $B 2 \mathrm{FeAl}$ were determined as functions of the Fe site vacancy concentration using several scattering techniques and were computed from first principles. Measurements at elevated temperature and pressure were performed to explore volume effects, test the usefulness of the quasiharmonic $(\mathrm{QH})$ approximation, and provide comparison for the first-principles calculations. The average temperature and pressure dependencies of phonons were consistent with the QH model. The decrease in specific volume associated with the introduction of vacancies causes a stiffening of the DOS that was captured well with the experimentally determined Grüneisen parameter. Features associated with vacancies in the DOS are not well explained by this model, however, especially in the gap between the acoustic and optic branches. First-principles calculations indicated that these modes are primarily associated with vibrations of $\mathrm{Al}$ atoms in the first-nearest-neighbor shell of the vacancy, with some vibration amplitude also involving the second-nearest-neighbor $\mathrm{Fe}$ atoms. At the vacancy concentrations of study, the phonon entropy of vacancy formation was found to be approximately $-1.7 k_{\mathrm{B}} /$ atom, about half as large and of opposite sign as the configurational entropy of vacancy formation.
\end{abstract}

DOI: 10.1103/PhysRevB.80.214303

PACS number(s): 63.20.-e, 65.40.gd, 61.72.-y, 61.05.fg

\section{INTRODUCTION}

Ordered intermetallic (B2) FeAl alloys are of technological interest as high-temperature structural materials due to their high resistance to corrosion, low density, and high strength. Unfortunately, the brittleness of these alloys at ambient temperatures hinders their use. An important characteristic of $B 2 \mathrm{FeAl}$ is its propensity to develop an abnormally high equilibrium vacancy concentration at high temperature, particularly at off-stoichiometric Al-rich concentrations where the predominant defect is the vacancy on the Fe site $\left(\mathrm{V}_{\mathrm{Fe}}\right) .{ }^{1,2}$ These large vacancy concentrations $x_{\mathrm{v}}$ can be retained at ambient temperature by quenching.

The concentrations of the different possible types of defects in FeAl depend strongly on both composition and temperature. Thermodynamic measurements and models have been used to rationalize the high vacancy concentrations in $B 2 \mathrm{FeAl} .{ }^{3-10}$ Vacancies increase the configurational entropy $S_{\mathrm{cf}}$, causing them to be favored at higher temperatures by lowering the Gibbs free energy, $G=H-T S$. The contribution of phonon entropy, $S_{\mathrm{ph}}$, to the vacancy formation free energy is often ignored, although $S_{\text {ph }}$ has been estimated to be large. ${ }^{11}$ Direct measurements allow for a better assessment of this important thermodynamic quantity.

Within the quasiharmonic $(\mathrm{QH})$ model, fractional changes in the phonon energies depend linearly on the fractional change in volume in proportion to the Grüneisen parameter $\gamma$. The volume of a crystal is determined by the pressure and temperature, which were varied in this experiment to explore effects of specific volume. The introduction of vacancies in $B 2 \mathrm{FeAl}$ results in a measurable decrease in the lattice parameter at ambient temperature. ${ }^{1}$ This decrease in lattice volume could result in an overall increase in the phonon frequencies, which would lead to a negative phonon entropy of vacancy formation. However, vacancies are also expected to locally distort the lattice in ways that should alter some phonon modes more than others, especially the high-frequency modes. The overall effect of vacancies on the phonon entropy is not obvious.

Here, we present results from experimental and computational studies of the effect of vacancies, temperature, and pressure on phonons in $B 2 \mathrm{FeAl}$. Inelastic neutron scattering (INS) was used to measure the dependence of the phonon density of states (DOS) on temperature and vacancy concentration. The ${ }^{57} \mathrm{Fe}$ phonon partial density of states (PDOS) was determined as a function of vacancy concentration and pressure up to $23 \mathrm{GPa}$ using nuclear resonant inelastic $\mathrm{x}$-ray scattering (NRIXS). The unit-cell volume was determined as a function of pressure up to $35 \mathrm{GPa}$ from energy dispersive $\mathrm{x}$-ray diffraction (EDXRD) and angle dispersive $\mathrm{x}$-ray diffraction (ADXRD). The phonon DOS was computed from first principles for the perfect $B 2$ structure, and in the $B 2$ structure with defects. We compare the results obtained with these different techniques and present a picture of the effect of vacancies on the phonons and phonon entropy of $B 2 \mathrm{FeAl}$. 


\section{INELASTIC NEUTRON SCATTERING}

\section{A. Materials preparation and characterization}

Large ingots of FeAl were vacuum cast at the Oak Ridge National Laboratory. The ingots were sliced using electric discharge machining into 1 -mm-thick pieces. They were sealed in quartz ampoules under high-purity argon and divided into three different batches. Each batch was annealed at a different temperature to form different equilibrium concentrations of vacancies. The samples were quenched into iced brine from the annealing temperatures $\left(T_{\mathrm{Q}}\right)$ of 823 , 1150 , and $1373 \mathrm{~K}$.

The $\mathrm{Fe}$ vacancy concentrations were estimated to be $0.5 \%, 1.8 \%$, and $3.1 \%$ for $T_{\mathrm{Q}}$ of 823,1150 , and $1373 \mathrm{~K}$, respectively, as follows. The density $\rho$ of each sample was measured by gravimetric analysis using ethanol as the flotation medium. Approximately 22 pieces from each sample were measured to obtain $\rho$ in Table I. X-ray diffraction patterns from all samples were acquired using an Inel CPS-120 diffractometer with Co $K \alpha$ radiation and a position-sensitive detector. A B2 crystal structure was confirmed, and lattice parameters were obtained from the XRD data. The densities and lattice parameters were used to determine the vacancy concentrations, following the method of Simmons and Balluffi $^{12}$ (adapted for density $\rho$ rather than length $L$ ),

$$
\Delta x_{\mathrm{v}} \approx 3\left(\frac{\Delta L}{L}-\frac{\Delta a}{a}\right) \approx\left(\frac{-\rho}{\Delta \rho}-1\right)^{-1}-\frac{3 \Delta a}{a} .
$$

Equation (1) gives the vacancy concentration difference between two samples. Measurements indicate that $x_{\mathrm{v}} \approx 0.5 \%$ at $823 \mathrm{~K} .{ }^{1}$ Table I presents the absolute vacancy concentrations, $x_{\mathrm{v}}=\Delta x_{\mathrm{v}}+0.5 \%$.

Six pieces were taken from the ingot for electron microprobe measurements. From 22 measurements of local chemical composition, the average alloy concentration was $50.3 \pm 0.2$ at. $\% \mathrm{Fe}$ and $49.7 \pm 0.2$ at. $\% \mathrm{Al}$. Trace amounts of Ni were also detected. We found the samples to have good composition homogeneity.

\section{B. Neutron-scattering experimental procedure}

Inelastic neutron-scattering measurements were performed with the low-resolution medium energy chopper spectrometer at the Intense Pulsed Neutron Source of the Argonne National Laboratory. Samples were mounted in thin-wall aluminum cans of area $7 \times 10 \mathrm{~cm}^{2}$. The flat sample packages were tilted at a $45^{\circ}$ angle off the direction of the incident beam to minimize self-shielding. In this arrangement, the $1-\mathrm{mm}$-thick pieces scattered on average $10 \%$ of the incident neutrons. All measurements were performed with an incident beam of monochromatic neutrons with a nominal energy of $60 \mathrm{meV}$. Data were collected at temperatures from 10 to $773 \mathrm{~K}$, using a closed-cycle He refrigerator for cooling and a custom low-background furnace for heating. Measurements were performed on the empty sample container at all temperatures under the same conditions.

The raw data of intensity versus time of flight in the different detector tubes were processed into intensity versus energy, and then converted into a "neutron-weighted" phonon density of states. The raw data were corrected for detector efficiency using a white beam spectrum from a vanadium plate and were normalized by the integrated incident flux on the sample. An average of the counts at the longest times of flight was taken as an estimate of the time-of-flightindependent background and subtracted from all data. The scattering from the empty holder and sample environment equipment was then subtracted from the data from the samples. The low-angle data (angles less than approximately $20^{\circ}$ ) were neglected to minimize possible contributions from magnetic scattering. After the removal of an elastic peak centered about $0 \mathrm{meV}$, the data below $8 \mathrm{meV}$ were truncated and approximated by a straight line, as expected in the continuum limit. Corrections were then made for thermal factors and multiphonon scattering (two- through five-phonon processes), using an iterative procedure in the incoherent approximation, with some accounting for multiple scattering. ${ }^{13}$

This procedure produces a neutron-weighted phonon DOS, because the cross sections for the scattering of thermal neutrons by $\mathrm{Fe}$ and $\mathrm{Al}$ nuclei are different. The scattering

TABLE I. Measured properties of quenched FeAl alloys at $300 \mathrm{~K}$.

\begin{tabular}{|c|c|c|c|c|c|c|c|c|}
\hline Pressure & $\begin{array}{l}T_{\mathrm{Q}} \\
(\mathrm{K})\end{array}$ & $\begin{array}{c}\rho \\
\left(\mathrm{g} / \mathrm{cm}^{3}\right)\end{array}$ & $\begin{array}{l}a \\
(\AA)\end{array}$ & $\begin{array}{l}x_{\mathrm{v}} \\
(\%)\end{array}$ & $\begin{array}{c}S_{\mathrm{ph}} \\
\left(k_{\mathrm{B}} / \text { atom }\right)\end{array}$ & $\begin{array}{c}\langle E\rangle \\
(\mathrm{meV})\end{array}$ & $\begin{array}{c}S_{\mathrm{ph}, \mathrm{Fe}} \\
{\left[k_{\mathrm{B}} /(\mathrm{Fe} \text { atom })\right]}\end{array}$ & $\begin{array}{c}\langle E\rangle_{\mathrm{Fe}} \\
(\mathrm{meV})\end{array}$ \\
\hline \multicolumn{9}{|l|}{ INS } \\
\hline Ambient & 823 & $5.57 \pm 0.03$ & $2.9168 \pm 0.0005$ & 0.5 & $3.055 \pm 0.013$ & $29.6 \pm 0.2$ & & \\
\hline Ambient & 1150 & $5.5 \pm 0.03$ & $2.9161 \pm 0.0005$ & 1.8 & $3.006 \pm 0.013$ & $30.2 \pm 0.2$ & & \\
\hline Ambient & 1373 & $5.48 \pm 0.01$ & $2.9079 \pm 0.0005$ & 3.1 & $3.010 \pm 0.013$ & $30.2 \pm 0.2$ & & \\
\hline \multicolumn{9}{|l|}{ NRIXS } \\
\hline Ambient & 773 & & $2.9130 \pm 0.0005$ & & & & $3.681 \pm 0.016$ & $23.2 \pm 0.1$ \\
\hline Ambient & 1273 & & $2.9037 \pm 0.0005$ & & & & $3.640 \pm 0.014$ & $23.7 \pm 0.1$ \\
\hline \multicolumn{9}{|l|}{ NRIXS } \\
\hline $23.0 \mathrm{GPa}$ & 773 & & $2.813 \pm 0.006$ & & & & $2.926 \pm 0.028$ & $30.37 \pm 0.1$ \\
\hline $23.0 \mathrm{GPa}$ & 1273 & & $2.812 \pm 0.006$ & & & & $2.875 \pm 0.025$ & $30.83 \pm 0.1$ \\
\hline
\end{tabular}




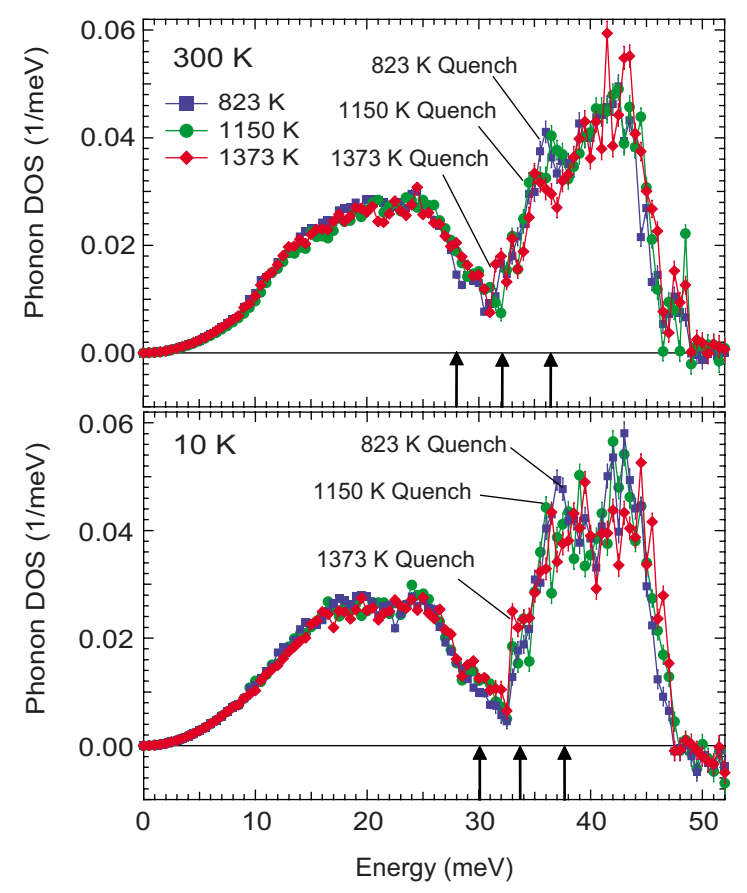

FIG. 1. (Color online) Phonon DOS of FeAl quenched from different temperatures, corrected for the neutron weighting, measured at 10 and $300 \mathrm{~K}$.

weight ratio $\mathrm{Fe}: \mathrm{Al}$ of $3.74: 1$ is quite large, requiring a neutron-weight correction. Fortunately, a neutron-weight correction is possible because $B 2 \mathrm{FeAl}$ is ordered with a two-atom basis. This system has both acoustic and optical modes, and the total number of modes is divided equally between the optical and acoustic branches. For $B 2 \mathrm{FeAl}$ the acoustic and optical branches are well separated. ${ }^{14}$ It is therefore straightforward to correct the data such that there are equal numbers of acoustic and optical modes in the DOS. The boundary between the acoustic and optic modes was chosen as the minimum observed around $30 \mathrm{meV}$ in the phonon DOS, guided by our first-principles calculations (Sec. IV).

\section{Neutron-scattering results}

Figure 1 presents the phonon DOS of FeAl quenched from different temperatures, measured at 10 and $300 \mathrm{~K}$. The phonon DOS is composed of two parts in all cases: the acoustic phonons contribute broad spectral intensity extending up to about $31 \mathrm{meV}$, while the optical phonons contribute two sharper peaks centered at 36 and $42 \mathrm{meV}$. The phonon cutoff is sharp and located at about $48 \mathrm{meV}$. The curves in Fig. 1 show that the introduction of a few percent of vacancies by quenching has a small effect on the phonon DOS of $B 2 \mathrm{FeAl}$; however, we do observe suppression of the number of modes in the lower optic peak, around $36 \mathrm{meV}$, and a concurrent slight stiffening (increase in energy) in the position of the phonon cutoff of $\sim 1 \mathrm{meV}$. Also, we observe higher shoulders on either side of the acoustic-optic gap in the samples with more vacancies. These features are highlighted by the upward arrows on the energy axis for both the 10 and $300 \mathrm{~K}$ plots. In the $10 \mathrm{~K}$ measurement, a peak can be

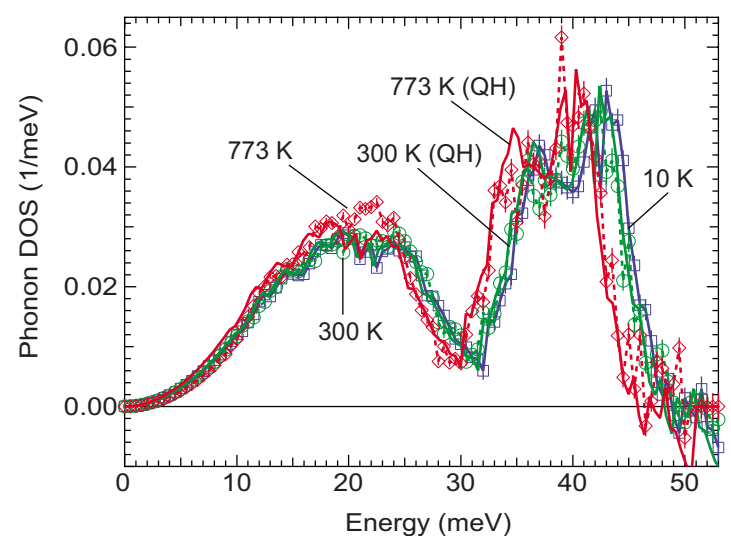

FIG. 2. (Color online) Phonon DOS of FeAl with low vacancy concentration (sample quenched from $823 \mathrm{~K}$ ) measured by INS at different temperatures (dashed lines and markers). Phonon DOS curves representing the effect of the change in volume from 10 to $300 \mathrm{~K}$ and $773 \mathrm{~K}$ were calculated from the measured $10 \mathrm{~K}$ DOS, using a quasiharmonic analysis [Eq. (2)] (see text). These calculated curves (solid lines) are designated "QH."

seen at $34 \mathrm{meV}$ in the sample with more vacancies. A broader peak is also seen around $30 \mathrm{meV}$. Although the vacancies themselves do not contribute to the phonon DOS, they can affect the vibrations of neighboring atoms, and we explain below how the peaks developing on either side of the gap can be related to the vibrations of first-nearest-neighbor $(1 \mathrm{NN}) \mathrm{Al}$ atoms around vacancies on the $\mathrm{Fe}$ sublattice.

The phonon DOS of FeAl with low vacancy concentration was measured at $773 \mathrm{~K}$. At this temperature there is a thermal equilibrium concentration of vacancies similar to the material quenched from $823 \mathrm{~K}$. Results for the phonon DOS (corrected for neutron weighting as described above) are shown in Fig. 2. The phonon DOS shows a definite softening with increasing temperature. The softening affects the whole phonon DOS and appears approximately proportional to the phonon energy. This behavior is expected as the dilation of the crystal lattice at elevated temperatures leads to a weakening of interatomic force constants.

\section{Quasiharmonic model}

The temperature dependence of the softening can be interpreted with the quasiharmonic model, according to which the fractional changes in the phonon energy levels depends linearly on the fractional change in volume,

$$
\frac{\Delta E}{E}=-\gamma \frac{\Delta V}{V},
$$

where the thermodynamic Grüneisen parameter (average over all phonon modes) is given by

$$
\gamma=\frac{3 \alpha V B_{S}}{C_{P}}
$$

with $\alpha$ is the linear coefficient of thermal expansion, $V$ is the specific volume, $B_{S}$ is the isentropic bulk modulus, and $C_{P}$ is the constant-pressure heat capacity. ${ }^{15}$ Using values from the 
literature for $C_{P},{ }^{16} \alpha,{ }^{17}$ and $B_{S}(148 \mathrm{GPa}),{ }^{18,19}$ and our experimental value for $V\left(24.8 \AA^{3}\right.$ per unit cell $)$ we obtain a Grüneisen parameter $\gamma=2.0$ at $300 \mathrm{~K}$. The high-pressure measurements presented in Sec. III C give $\gamma_{\mathrm{Fe}}=1.73 \pm 0.04$, which is confirmed by the calculations presented in Sec. IV C.

Using the experimentally determined Grüneisen parameter $(\gamma=1.73)$ we shifted the $10 \mathrm{~K}$ DOS to the 300 and $773 \mathrm{~K}$ volumes following Eq. (2) (for the $823 \mathrm{~K}$ quench). This is shown in Fig. 2 as solid lines. For the high-temperature measurements ( 300 and $773 \mathrm{~K}$ ) we used the lattice volume from the results of neutron-diffraction measurements presented by Kogachi et al. ${ }^{2}\left(a_{300 \mathrm{~K}}=2.910 \AA\right.$ and $\left.a_{773 \mathrm{~K}}=2.937 \AA\right)$. The $10 \mathrm{~K}$ lattice parameter $\left(a_{10} \mathrm{~K}=2.902 \AA\right)$ was determined from thermal expansion data taken from 120 to $300 \mathrm{~K}$ and $C_{\mathrm{V}}$ data taken down to $10 \mathrm{~K}$ (Ref. 20) by scaling $C_{\mathrm{V}}$ to $\alpha$ at $120 \mathrm{~K}$. The similarity between the DOS measured at 300 and $773 \mathrm{~K}$ and the DOS calculated using the quasiharmonic analysis shows that phonons are quasiharmonic at temperatures up to $773 \mathrm{~K}$ for $\mathrm{B} 2 \mathrm{FeAl}$.

\section{E. Phonon entropy of vacancy formation}

The phonon entropy $S_{\mathrm{ph}}$ is obtained from the phonon DOS $g(E) \mathrm{as}^{21}$

$$
S_{\mathrm{ph}}=3 k_{\mathrm{B}} \int d E g(E)[(n+1) \ln (n+1)-n \ln n],
$$

where $n=\left\{\exp \left[E /\left(k_{\mathrm{B}} T\right)\right]-1\right\}^{-1}$. Results are listed in Table I.

The phonon entropies in Table I for different vacancy concentrations can be used to determine the phonon entropy of vacancy formation $\Delta S_{\mathrm{ph}}^{\mathrm{V}_{\mathrm{Fe}}}$ by appropriate conversion,

$$
\Delta S_{\mathrm{ph}}^{\mathrm{V}_{\mathrm{Fe}}}=\Delta S_{\mathrm{ph}} \frac{N_{\mathrm{at}}}{\Delta N_{\mathrm{v}}} .
$$

The ratio $\Delta N_{\mathrm{v}} / N_{\text {at }}$ gives the relative change in the number of vacancies per number of atoms,

$$
\frac{N_{\mathrm{at}}}{\Delta N_{\mathrm{v}}}=\left[\frac{x_{\mathrm{v}, 2}}{1-x_{\mathrm{v}, 2}}-\frac{x_{\mathrm{v}, 1}}{1-x_{\mathrm{v}, 1}}\right]^{-1},
$$

where $x_{\mathrm{v}, 1}$ and $x_{\mathrm{v}, 2}$ are the vacancy concentrations for two different samples. In the dilute limit $\left(1-x_{\mathrm{v}, 1}\right) \simeq\left(1-x_{\mathrm{v}, 2}\right) \simeq 1$, so that $\Delta S_{\mathrm{ph}}=\Delta x_{\mathrm{v}} \Delta S_{\mathrm{ph}}^{\mathrm{V}_{\mathrm{Fe}}}$ and the slope of a plot of $\Delta S_{\mathrm{ph}}$ versus $x_{\mathrm{v}}$ gives $\Delta S_{\mathrm{ph}}^{\mathrm{V}_{\mathrm{Fe}}}$. A linear least-squares fit gives a phonon entropy per vacancy of $-(1.7 \pm 0.7) k_{\mathrm{B}}$ /vacancy.

The temperature dependence of the DOS shows that FeAl is well described by the $\mathrm{QH}$ model. The phonon entropy of vacancy formation was calculated using the $\mathrm{QH}$ model as follows. The $823 \mathrm{~K}$ quench data $(a=2.9168 \AA)$ shown in Fig. 1 measured at $300 \mathrm{~K}$ was shifted in energy according to Eq. (2) $(\gamma=1.73)$ to the volume of the $1373 \mathrm{~K}$ quench sample $(a=2.9079 \AA)$. This yields a phonon entropy of $3.013 k_{\mathrm{B}}$ /atom, similar to the measured $S_{\mathrm{ph}}$ for the $1373 \mathrm{~K}$ quench sample of $3.010 k_{\mathrm{B}} /$ atom. This application of the $\mathrm{QH}$ model to the phonon DOS gives $\Delta S_{\mathrm{ph}}^{\mathrm{V}_{\mathrm{Fe}}}=-1.6 k_{\mathrm{B}} /$ vacancy, within the error bars of our measurement.

From our neutron-scattering measurements, the phonon entropy of vacancy formation on the Fe sublattice appears similar in magnitude to its configurational counterpart $\Delta S_{\mathrm{cf}}^{\mathrm{V} F e}$. Assuming that energetics constrain the vacancies to the $\mathrm{Fe}$ sublattice, and there are no correlations between vacancies, the configurational entropy from the number of ways the vacancies can be distributed on the Fe sublattice is

$$
S_{\mathrm{cf}}^{\mathrm{V}_{\mathrm{Fe}}}\left(x_{\mathrm{v}}\right)=\frac{-k_{\mathrm{B}}}{2}\left[2 x_{\mathrm{v}} \ln 2 x_{\mathrm{v}}+\left(1-2 x_{\mathrm{v}}\right) \ln \left(1-2 x_{\mathrm{v}}\right)\right],
$$

where the factor of $1 / 2$ converts the units from $\left[k_{\mathrm{B}} / \mathrm{Fe}\right.$ atom $]$ to $\left[k_{\mathrm{B}} /\right.$ atom $]$. Taking the discrete difference in the configurational entropy between the samples with $0.5 \%$ and $3.1 \%$ vacancies and converting using Eq. (6) gives a value of $\Delta S_{\mathrm{cf}}^{\mathrm{V} F e}=3.392 k_{\mathrm{B}} /$ vacancy, making the magnitude of phonon entropy of vacancy formation about half of the configurational entropy of vacancy formation.

\section{HIGH-PRESSURE MEASUREMENTS}

\section{A. Sample preparation and characterization}

For the diamond anvil cell (DAC) measurements a single ingot of $B 2$ ordered ${ }^{57} \mathrm{FeAl}$, with an excess of $\mathrm{Al}$, was prepared by arc melting ${ }^{57} \mathrm{Fe}$ of $95.38 \%$ isotopic enrichment and natural aluminum of $99.98 \%$ purity under an argon atmosphere. The sample was annealed at $1373 \mathrm{~K}$ for $12 \mathrm{~h}$ under vacuum to ensure homogeneity and then sectioned with a diamond saw. A mass loss of approximately $1 \%$ was detected and attributed to $\mathrm{Al}$ loss, and electron microprobe measurements established a final composition of ${ }^{57} \mathrm{Fe}_{0.492} \mathrm{Al}_{0.508}$. The samples were pulverized in preparation for loading into a DAC, with particle sizes ranging from 10 to $200 \mu \mathrm{m}$. The powders were annealed under vacuum at 773 and $1273 \mathrm{~K}$ for 7 days and $1 \mathrm{~h}$, respectively, followed by a quench into iced brine. The vacancy concentration $x_{\mathrm{v}}$ for the samples with different quench temperatures can be estimated from the literature $^{1,2}$ to be $x_{\mathrm{v}}(1273 \mathrm{~K}) \simeq 3-4 \%$ and $x_{\mathrm{v}}(773 \mathrm{~K})$ $\simeq 1 \%$. The difference in the lattice parameter between the 773 and $1273 \mathrm{~K}$ quench NRIXS samples reported in Table I is similar to the difference between the corresponding quench temperatures of the neutron-scattering samples. Therefore, the difference in the vacancy concentrations between the two NRIXS samples is assumed to be the same as for the neutron-scattering samples $\left(\Delta x_{\mathrm{v}}=2.6 \%\right)$.

\section{B. Elastic x-ray scattering}

High-pressure ADXRD and EDXRD were performed on samples from both quench temperatures at the National Synchrotron Light Source at the Brookhaven National Laboratory on beamline $\mathrm{X}-17 \mathrm{C}$. The samples were loaded into a Merill-Bassett-type DAC (Ref. 22) with diamonds having $500 \mu \mathrm{m}$ culets, silicon oil as the pressure medium, and 301 stainless steel as a gasket material. The pressure was calibrated with the ruby fluorescence technique. ${ }^{23}$ The $\mathrm{x}$-ray energy range for EDXRD was $5-100 \mathrm{keV}$ with a beam dimension of $0.01 \times 0.01 \mathrm{~mm}^{2}$ and the detector set at $2 \theta \simeq 24^{\circ}$. The angle and energy were calibrated prior to the experiment. For the ADXRD experiment a charge-coupled device detector was placed off center to the incident beam at a distance of $196 \mathrm{~mm}$ from the sample. The incident wavelength 


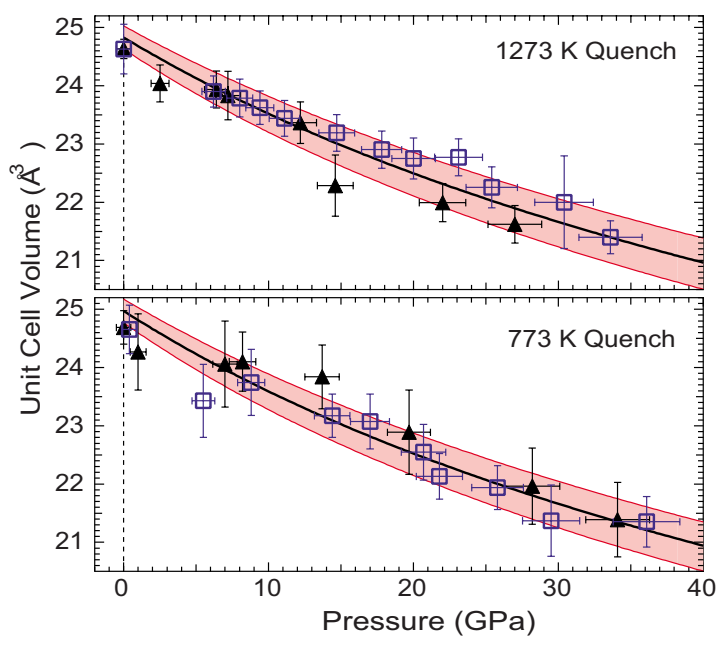

FIG. 3. (Color online) Pressure versus volume data determined from angle (solid triangles) and energy (open squares) dispersive $\mathrm{x}$-ray diffraction. The solid black line shows the fit to the data, and the shaded regions give the error on the fit.

was $0.4066 \AA$, with a beam dimension of $0.02 \times 0.02 \mathrm{~mm}^{2}$. The angle of tilt of the detector, distance from sample to detector, and the incident wavelength were calibrated prior to the experiment. The angular window of the DAC allowed for a minimum $d$ spacing of $\sim 1.5 \AA$. No structural phase transition was found over the pressure range of the experiment.

The pressure versus volume data from both EDXRD and ADXRD for the two different quench temperatures are shown in Fig. 3. The lattice volume determined from the data can be used to find the ambient pressure bulk modulus $B_{0}$ using the Murnaghan equation of state,

$$
V(P)=V_{0}\left[1+B_{0}^{\prime} \frac{P}{B_{0}}\right]^{-1 / B_{0}^{\prime}},
$$

where $V_{0}$ is the ambient pressure volume and $B_{0}^{\prime}$ is the pressure derivative of the bulk modulus. The value of $B_{0}^{\prime}$ is set to 4 (typical of cubic systems), so that $V_{0}$ and $B_{0}$ are the fitting parameters. The fits yield values of $B_{0}^{773 \mathrm{~K}}=157 \pm 15 \mathrm{GPa}$, $B_{0}^{1273 \mathrm{~K}}=165 \pm 17 \mathrm{GPa}, \quad V_{0}^{773 \mathrm{~K}}=24.97 \pm 0.20 \AA^{3}, \quad$ and $V_{0}^{1273 \mathrm{~K}}=24.83 \pm 0.20 \AA^{3}$. These values indicate that within experimental error there is no measurable difference in the bulk modulus between the two quench temperatures. Extrapolation of sound velocity measurements for ironaluminum alloy single crystals in the composition range from 4 to 40 at. \% aluminum at $300 \mathrm{~K}$ gives a value of $B_{0}$ $=148 \mathrm{GPa},{ }^{18,19}$ which is within the error bars of our result. The values of $V_{0}$ from the measurements are in good agreement with the values in the literature ${ }^{1,2}$ and in Table I.

\section{Nuclear resonant inelastic $x-$ ray scattering}

High-pressure NRIXS (Refs. 24-26) was performed at beamline 16ID-D at the Advanced Photon Source of the Argonne National Laboratory. The samples quenched from 773 and $1273 \mathrm{~K}$ were loaded into separate panoramic pistoncylinder-type DACs optimized for the use in NRIXS experiments. ${ }^{27}$ Beryllium was used as a gasket material to

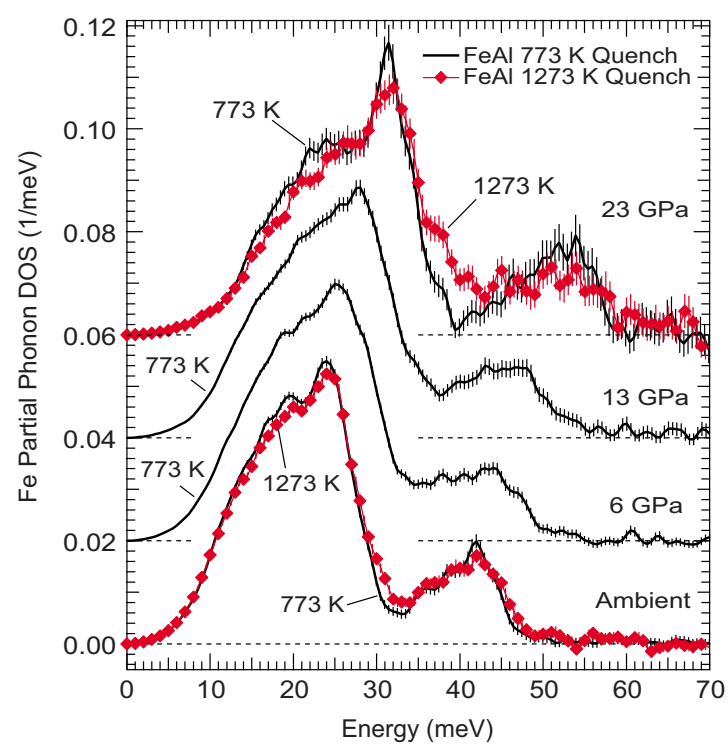

FIG. 4. (Color online) ${ }^{57} \mathrm{Fe}$ PDOS curves from NRIXS as a function of pressure for samples quenched from $773 \mathrm{~K}\left(x_{\mathrm{v}} \simeq 1 \%\right)$ and $1273 \mathrm{~K}\left(x_{\mathrm{v}} \simeq 3-4 \%\right)$. The PDOS curves are offset by integer multiples of $0.02 \mathrm{meV}^{-1}$.

allow transmission of the NRIXS signal, which was measured with three avalanche photodiode detectors positioned $90^{\circ}$ from the direction of the beam. The incident photon energy was tuned to $14.413 \mathrm{keV}$, the nuclear resonance energy of ${ }^{57} \mathrm{Fe}$. Data were collected in scans of incident photon energy from -80 to $+80 \mathrm{meV}$ from the resonant energy, with a monochromator resolution of $2.2 \mathrm{meV}$. For measurements at ambient pressure, each sample was placed on Kapton tape at a grazing angle to the incident photon beam.

The raw scattering data were summed and analyzed using the program PHOENIX, ${ }^{28}$ which calculates the ${ }^{57} \mathrm{Fe}$ PDOS, including the removal of the elastic peak and multiphonon corrections. The ${ }^{57} \mathrm{Fe}$ PDOS curves for the samples measured in this experiment are shown in Fig. 4. The ambient pressure Fe PDOS curves are similar to those obtained using $a b$ initio calculations (Sec. IV and Ref. 14).

With more vacancies, the Fe PDOS curves at ambient pressure show a measurable decrease in the number of modes in the energy range $15-25 \mathrm{meV}$, and an increase in modes in the energy range $28-32 \mathrm{meV}$. The sample with more vacancies also shows a slight increase in the phonon cutoff energy. This trend is consistent with the results of neutron scattering presented in Fig. 1. The shift of the phonon frequencies into the gap is larger at $23 \mathrm{GPa}$ than at ambient pressure.

The phonon entropy for the Fe PDOS is given in Table I in units of $k_{\mathrm{B}} /(\mathrm{Fe}$ atom $)$. Accounting for the concentration of $\mathrm{Fe}$ atoms and converting using Eq. (6) we determine the contribution of the Fe modes to the phonon entropy of vacancy formation to be $\Delta S_{\mathrm{ph}, \mathrm{Fe}}^{V_{\mathrm{Fe}}}=-(0.77 \pm 0.40) k_{\mathrm{B}} /$ vacancy.

The fits to the pressure versus volume data given in Sec. III B and the first moment of the PDOS curves in Fig. 4 provide the information necessary to determine the thermodynamic Grüneisen parameter of Eq. (2) for the Fe modes. The value obtained from the $773 \mathrm{~K}$ quench sample is $\gamma_{\mathrm{Fe}}$ 
$=1.73 \pm 0.04$. For the $1273 \mathrm{~K}$ quench sample $\gamma_{\mathrm{Fe}}$ $=1.68 \pm 0.05$.

The phonon entropy of vacancy formation for the $\mathrm{Fe}$ modes was calculated using the $\mathrm{QH}$ model as follows. The $773 \mathrm{~K}$ quench data $(a=2.9130 \AA)$ shown in Fig. 4 measured at ambient pressure was shifted in energy according to Eq. (2) $\left(\gamma_{\mathrm{Fe}}=1.73\right)$ to the volume of the $1273 \mathrm{~K}$ quench sample $(a=2.9037 \AA)$. This yields a phonon entropy of $3.637 k_{\mathrm{B}} /\left(\mathrm{Fe}\right.$ atom), similar to the measured $S_{\mathrm{ph}, \mathrm{Fe}}$ for the $1273 \mathrm{~K}$ quench sample of $3.640 \mathrm{k}_{\mathrm{B}}$ / atom. This application of the $\mathrm{QH}$ model to the Fe PDOS gives $\Delta S_{\mathrm{ph}, \mathrm{Fe}}^{\mathrm{V}_{\mathrm{Fe}}}$ $=-0.85 k_{\mathrm{B}} /$ vacancy, within the error bars of our measurement.

\section{FIRST-PRINCIPLES SIMULATIONS}

Density-functional theory (DFT) calculations of phonons in FeAl have been performed by Meyer et al. ${ }^{14}$ and these authors reported very good agreement with the experimental phonon dispersion. The computations of Meyer et al. ${ }^{14}$ were limited to the case of perfect $B 2 \mathrm{FeAl}$ with no defects, however. Here, we report on first-principles calculations of the structure and lattice dynamics of FeAl for the cases of both the perfect structure, and for $B 2 \mathrm{FeAl}$ with vacancies and antisites on the $\mathrm{Fe}$ and $\mathrm{Al}$ sublattices.

\section{A. Methods}

We used $2 \times 2 \times 2$ and $3 \times 3 \times 3$ supercells of the $B 2 \mathrm{FeAl}$ unit cell, containing respectively 16 and 54 atoms. To model the alloy with $\mathrm{Fe}$ vacancies, we used a $3 \times 3 \times 3$ supercell with the central $\mathrm{Fe}$ atom removed, representing a vacancy concentration of $1.85 \%$. Similarly, for an Al antisite defect, the central $\mathrm{Fe}$ atom was replaced with an $\mathrm{Al}$ atom, and vice versa for the Fe antisite.

DFT simulations were performed with the software VASP, using the projector augmented wave formalism. ${ }^{29-32}$ All calculations were performed with the Perdew-Burke-Ernzerhof1996 generalized gradient approximation as the exchangecorrelation functional. ${ }^{33}$ The total energy of $B 2$ FeAl was well converged with a Brillouin-zone sampling grid of 12 $\times 12 \times 12 k$ points, and the energy of the $3 \times 3 \times 3 \mathrm{FeAl}$ supercells was converged with $4 \times 4 \times 4$ grids. The energy cutoff was $268 \mathrm{eV}$, except for volume optimization (and relaxation calculation in the case of supercell with defects), for which a cutoff of $350 \mathrm{eV}$ was used. Calculations were performed as a function of lattice parameter to determine the bulk modulus $B$ and its pressure derivative $B^{\prime}$. For the supercell with defects, the positions of the ions were optimized at each volume. The resulting $E(V)$ curves were fit with a Birch-Murnaghan equation ${ }^{34}$ to extract $B$ and $B^{\prime}$.

We used the direct force method ${ }^{35-37}$ to compute the lattice dynamics of FeAl with and without point defects. The software PHONON ${ }^{38}$ generated a set of atomic displacements, allowing the determination of the interatomic force-constant tensor. The forces on ions in each configuration were computed with DFT (VASP) with the same settings as above.

The phonons for the perfect structure were calculated with both $2 \times 2 \times 2$ and $3 \times 3 \times 3$ supercells. The phonons for the
$3 \times 3 \times 3$ supercells with central defects were calculated with the same method, using the relaxed structures. For supercells without central defects, only two displacements had to be calculated, corresponding to the two types of atoms. For the supercells with central defects, a total of 12 inequivalent displacements had to be calculated for antisite defects and 11 displacements in the case of the Fe vacancy. The displacement amplitudes were set to $0.03 \AA$, in all cases. The calculated phonon DOS curves for the $2 \times 2 \times 2$ and $3 \times 3 \times 3$ supercells were very similar, and energies of the optical modes were in excellent agreement. The acoustic region of the DOS had a very similar shape in both cases, but a shift in energy was observed, as expected from the wavelength cutoff imposed by the smaller $2 \times 2 \times 2$ supercell. However, the very good overall agreement indicates that the phonon DOS is largely converged with a $3 \times 3 \times 3$ supercell.

\section{B. Structure optimization}

The optimized lattice parameters and bulk modulus of the supercells are listed in Table II. Our result for the equilibrium lattice parameter of the perfect structure is in good agreement with the low-temperature lattice parameter (Sec. II D), being only $1.1 \%$ smaller than the value of $2.902 \AA$. The optimized lattice parameter is $1.6 \%$ smaller than the experimental value of $2.9168 \AA$ (at $300 \mathrm{~K}$ ). The result of our calculation for bulk modulus is somewhat larger than the experimental value, which is expected from the smaller computed equilibrium lattice parameter. The computed values for the bulk modulus derivative, $4.1 \leq B^{\prime} \leq 4.4$, are consistent with the value used in the fits of experimental $V(P)$ curves. For the perfect $B 2$ structure, using the experimental value of bulk modulus $B=148 \mathrm{GPa}$ (for the low vacancy concentration), we find that the pressure that would result in lattice parameter decrease of $1.6 \%$ is $4.3 \mathrm{GPa}$. Using $B^{\prime}$ $=4.4$, this leads to an overestimate of the bulk modulus by 19 $\mathrm{GPa}$, accounting for the discrepancy between the experimental and computed values.

The point defects had only a small effect on the computed lattice parameter of the $3 \times 3 \times 3$ supercells. In particular, the first-principles calculations predict a very small overall contraction upon introduction of $1.85 \%$ Fe vacancies (less than a $10^{-3}$ relative change). The magnitude of this change is in good agreement with the measured change in lattice parameter between the samples quenched from 823 and $1150 \mathrm{~K}$. The computed bulk modulus for the structure with Fe vacancies is about 4\% smaller than for the perfect structure, whereas our measurements detected no change in $B$, within experimental uncertainty. The DFT computations predict a similarly small decrease in $B$ in the case of $1.85 \% \mathrm{Al}$ antisite defects, and almost no change in the case of $1.85 \% \mathrm{Fe}$ antisites.

Some distances between the central atom or defect and the atoms in the $n$ th-nearest-neighbor $(n \mathrm{NN})$ shells are listed in Table II. The optimized structures, with displacements exaggerated by a factor of 4, are shown in Fig. 5. This figure and Table II show that the introduction of a vacancy on the Fe sublattice causes a contraction of the $1 \mathrm{NN} \mathrm{Al}$ atoms toward the vacancy, while the Fe atoms in the $2 \mathrm{NN}$ around the 
TABLE II. Results from structure optimization with VASP: $a$ is the lattice parameter, $B$ is the bulk modulus, $B^{\prime}$ its pressure derivative, and $L_{n}$ is the $n$th nearest-neighbor distance from the central atom (or vacancy) in the $3 \times 3 \times 3$ supercell. Experimental and literature values are at $300 \mathrm{~K}$.

\begin{tabular}{|c|c|c|c|c|c|c|c|}
\hline & $\begin{array}{c}a \\
(\AA)\end{array}$ & $\begin{array}{c}B \\
(\mathrm{GPa})\end{array}$ & $B^{\prime}$ & $\begin{array}{c}L_{1}(\AA) \\
L_{1} / a\end{array}$ & $\begin{array}{c}L_{2}(\AA) \\
L_{2} / a\end{array}$ & $\begin{array}{c}L_{3}(\AA) \\
L_{3} / a\end{array}$ & $\begin{array}{c}L_{4}(\AA) \\
L_{4} / a\end{array}$ \\
\hline Expt. & 2.9168 & $157 \pm 15$ & & & & & \\
\hline Lit. $^{\mathrm{a}}$ & 2.910 & 148 & & & & & \\
\hline $\mathrm{Fe}_{27} \mathrm{Al}_{27}$ & 2.870 & 181 & 4.4 & 2.485 & 2.870 & 4.060 & 4.759 \\
\hline No defect & & & & 0.866 & 1.000 & 1.414 & 1.658 \\
\hline $\mathrm{Fe}_{26} \mathrm{Al}_{27}$ & 2.868 & 174 & 4.3 & 2.442 & 2.912 & 4.02 & 4.77 \\
\hline $\mathrm{V}_{\mathrm{Fe}}$ & & & & 0.852 & 1.015 & 1.401 & 1.664 \\
\hline $\mathrm{Fe}_{26} \mathrm{Al}_{28}$ & 2.880 & 173 & 4.1 & 2.624 & 2.810 & 4.070 & 4.770 \\
\hline $\mathrm{Al}_{\mathrm{Fe}}$ & & & & 0.911 & 0.977 & 1.415 & 1.657 \\
\hline $\mathrm{Fe}_{28} \mathrm{Al}_{26}$ & 2.865 & 183 & 4.4 & 2.429 & 2.871 & 4.045 & 4.753 \\
\hline $\mathrm{Fe}_{\mathrm{Al}}$ & & & & 0.848 & 1.002 & 1.412 & 1.659 \\
\hline
\end{tabular}

${ }^{\mathrm{a} R e f e r e n c e s} 2$ and 18.

vacancy move away from the vacancy. In the case of the $\mathrm{Al}$ antisite $\left(\mathrm{Fe}_{26} \mathrm{Al}_{28}\right)$, the $\mathrm{Al}$ atoms in the $1 \mathrm{NN}$ shell are pushed away from the defect, and Fe atoms in the $2 \mathrm{NN}$ shell come closer to it. On the other hand, the Fe antisite binds strongly to $1 \mathrm{NN} \mathrm{Fe}$ atoms, leading to a contraction of this shell and a small expansion of the $2 \mathrm{NN}$ shell of $\mathrm{Al}$ atoms. The computed strains are relatively small in the $3 \mathrm{NN}$ and $4 \mathrm{NN}$ shells in all cases.

\section{Effect of compression on the phonon DOS}

The phonon DOS of the perfect structure was computed for reduced volumes. The $a b$ initio phonon frequencies are larger than the experimental values by only about $6 \%$, consistent with the computed lattice parameter being smaller than the experimental value by $1.6 \%$. This corresponds to an experimental pressure of approximately $5 \mathrm{GPa}$, as was discussed above. Figure 6 shows the Fe phonon partial DOS for perfect $B 2 \mathrm{FeAl}(2 \times 2 \times 2$ supercell) for increasing compressions (decreasing lattice parameters). The phonon DOS systematically stiffens with increasing compression, as expected in the quasiharmonic model, and as observed experimentally (see Fig. 4). Also, the magnitudes of the shifts in the phonon energies at different pressures are in good agreement with our measurements. The calculated total phonon

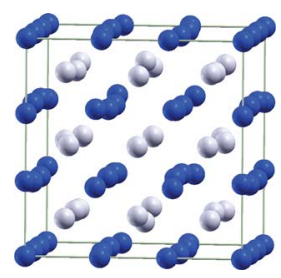

(a)

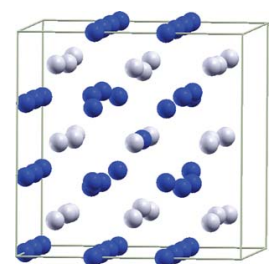

(b)

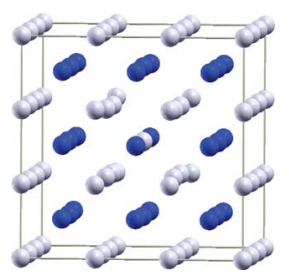

(c)
FIG. 5. (Color online) Relaxed $3 \times 3 \times 3$ supercells with central defects, calculated with VASP. The displacements from the perfect structure are exaggerated by a factor of 4 for visualization. Light atoms (gray) are Fe; dark atoms (blue) are Al. Left: Fe vacancy. Center: Al antisite. Right: Fe antisite.
DOS and the Al partial DOS (not shown) follow the same trend. The Grüneisen parameter of $B 2 \mathrm{FeAl}$ was obtained from the first moment of the computed DOS, $\langle E\rangle$ : $\gamma$ $=-d \ln \langle E\rangle / d \ln V$, and component-specific Grüneisen parameters $\gamma_{i}=-d \ln \left\langle E_{i}\right\rangle / d \ln V$, where $i$ stands for either Fe or $\mathrm{Al}$ [these equations are equivalent to Eq. (2)]. Using a linear approximation around the theoretical equilibrium volume, we find $\gamma=1.76, \gamma_{\mathrm{Fe}}=1.86$, and $\gamma_{\mathrm{Al}}=1.69$. The theoretical value for $\mathrm{Fe}$ modes is in fairly good agreement with our NRIXS result of 1.73. It is a little larger, however, as expected from the overestimate in the computed bulk modulus, as discussed previously.

\section{Effect of defects on the phonon DOS}

The calculated phonon DOS curves for $3 \times 3 \times 3 \mathrm{~B} 2 \mathrm{FeAl}$ with and without defects are shown in Fig. 7. A vacancy on the Fe sublattice (1 in $27 \mathrm{Fe}$ atoms vacant, or 1 in 54 atoms overall, corresponding to a concentration of $1.85 \%$ ) leads to a filling of the gap between the acoustic and optical modes

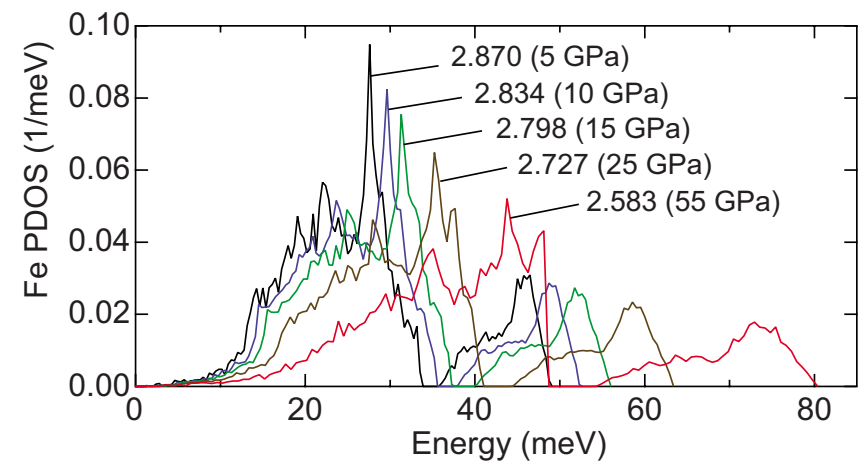

FIG. 6. (Color online) Results of first-principles phonon DOS calculations for FeAl $2 \times 2 \times 2$ supercell with different lattice parameters for the Fe partial phonon DOS. All the curves are normalized to unity. The labels correspond to the value of lattice parameter in $\AA$. The pressures in parentheses are approximate experimental values based on the fit to the $P(V)$ data of Fig. 3 . 


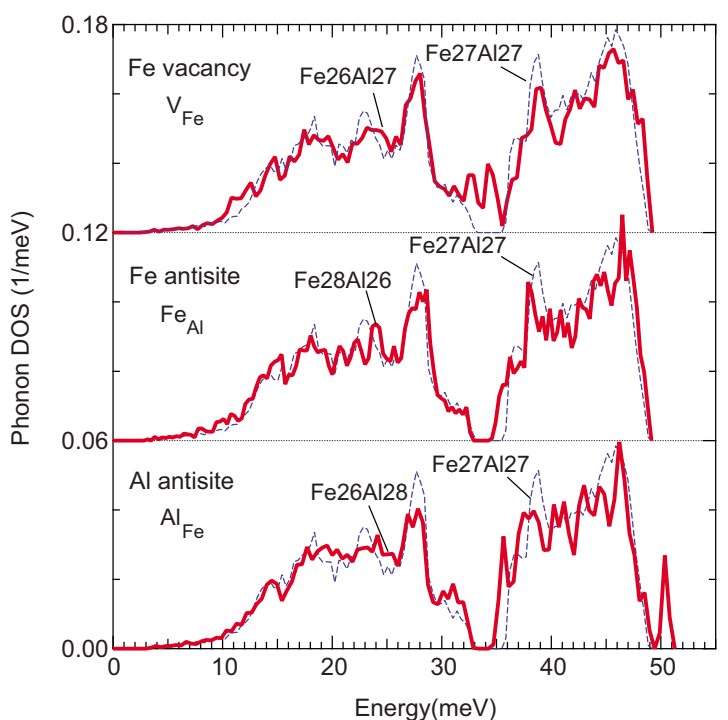

FIG. 7. (Color online) Phonon DOS for FeAl $3 \times 3 \times 3$ supercells for the perfect structure (dashed curves) and with central defects (thick solid curves), calculated with VASP.

(from 33 to $35 \mathrm{meV}$ ). Two peaks appear in the gap: one just above the cutoff energy for the acoustic modes in the perfect structure and one just below the band minimum for the optical modes. This behavior matches very well the observed trend in the phonon DOS measurements (Sec. II). On the other hand, the $\mathrm{Fe}$ and $\mathrm{Al}$ antisite defects produce no modes in the acoustic-optic gap. In the case of the Fe antisite (Fe atom on $\mathrm{Al}$ site), the phonon DOS presents a sharp peak (of limited spectral weight) at negative energies (around $-7 \mathrm{meV}$, not shown in Fig. 7), associated with motions of the $\mathrm{Fe}$ antisite atom. Although nonphysical in a strict sense, negative frequencies represent vibration modes for which the structure is mechanically unstable. No instability was found for the $\mathrm{Al}$ antisite defect.

Figures 7 and 8 show that the new gap modes induced by the Fe vacancy are primarily associated with vibrations of $\mathrm{Al}$ atoms. The $\mathrm{Al}$ atom gap modes are $1 \mathrm{NN}$ atoms to the vacancy, with some vibration amplitude also involving the $\mathrm{Fe}$ atoms that are $2 \mathrm{NN}$ to the vacancy. The atoms further from the vacancy make only minor contributions. Evidently, the Al atoms neighboring the vacancy can explore a larger free volume in their vibrations and tend to vibrate at lower frequencies.

For comparison, Fig. 9 shows the experimental and calculated phonon DOS curves scaled to the same experimental volume $(a=2.9168 \AA)$. The calculated phonon DOS curves are in good agreement with our inelastic neutron-scattering measurements on FeAl. This is encouraging for both the first-principles phonon calculations, and also for the neutronweight correction of the experimental data. Having all been scaled to the same volume, the differences in the curves show the phonon features due to vacancies.

\section{DISCUSSION}

The calculations and measurements indicate that vacancies alter the forces in $\mathrm{FeAl}$ in two distinct ways. First, the

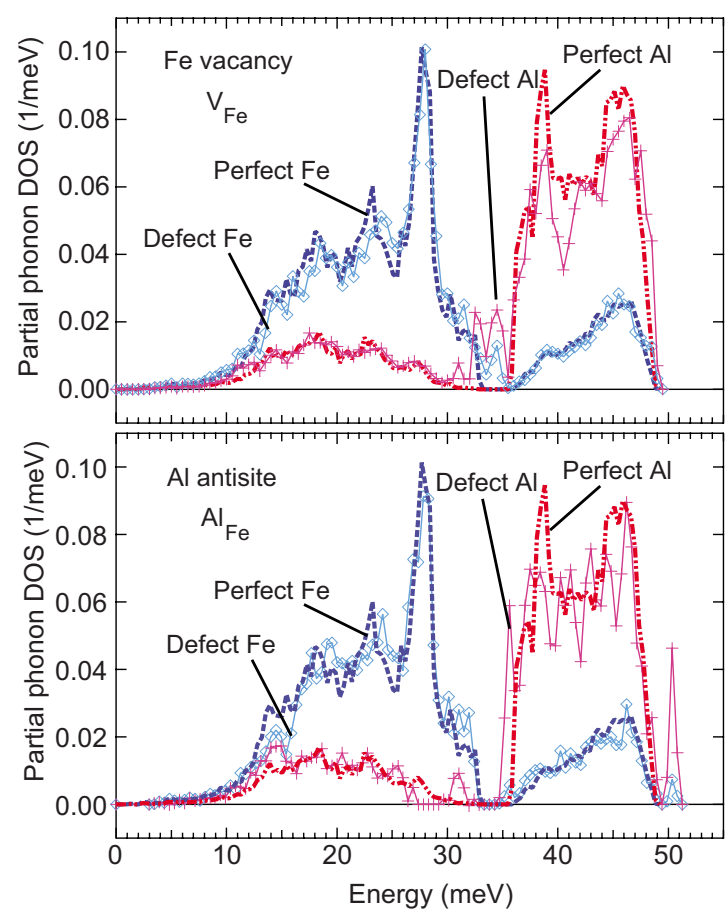

FIG. 8. (Color online) Top: partial phonon DOS for FeAl 3 $\times 3 \times 3$ supercell corresponding to perfect structure and structure with central Fe vacancy. Bottom: same for $3 \times 3 \times 3$ structure with central $\mathrm{Al}$ antisite on Fe sublattice.

introduction of vacancies reduces the lattice parameter and causes an overall increase in atomic forces. This induces a positive shift in energy of all phonon modes consistent with the Grüneisen parameter. Second, there is a local distortion of the unit cell in the vicinity of the vacancy that changes the local forces of the surrounding atoms. This increases the number of modes in the gap between the acoustic and optical phonon branches.

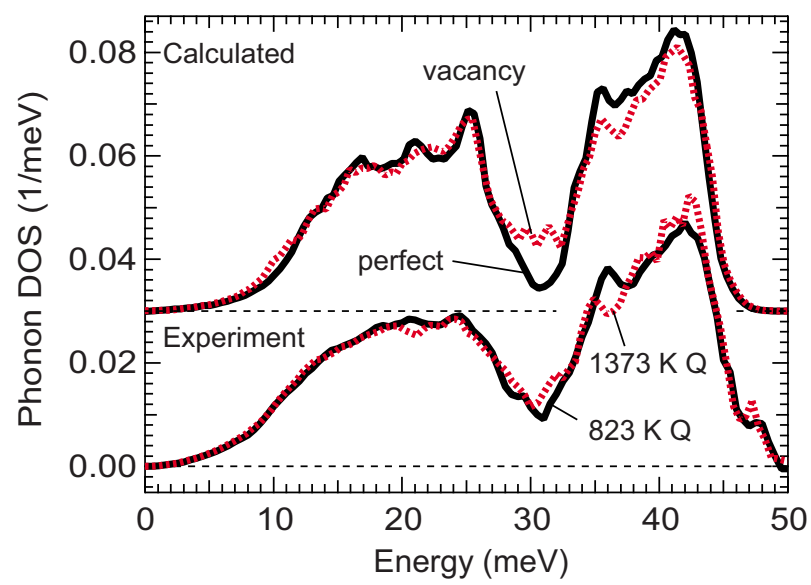

FIG. 9. (Color online) Phonon DOS curves shifted to the $823 \mathrm{~K}$ quench volume (at $300 \mathrm{~K}, a=2.9168 \AA$ ) following Eq. (2) with $\gamma$ $=1.73$ using the values of the volume given in Tables I and II. The experimental data (bottom curves) are averaged over $1 \mathrm{meV}$ to reduce noise. The calculated data (top curves) are convolved with the experimental resolution function. The DOSs with higher vacancy concentrations are given by dashed lines. 
The changes in the DOS lead to a measured phonon entropy of vacancy formation of $-(1.7 \pm 0.7) k_{\mathrm{B}}$ /vacancy. For the Fe modes the phonon partial entropy of Fe vacancy formation is measured to be $\Delta S_{\mathrm{ph}, \mathrm{Fe}}^{\mathrm{V}_{\mathrm{Fe}}}=-(0.77 \pm 0.40)$ $k_{\mathrm{B}}$ /vacancy. The $\mathrm{QH}$ model indicates similar values. The change in the configurational entropy per vacancy is positive and was estimated to be $3.4 k_{\mathrm{B}}$ / vacancy, stabilizing vacancies at higher temperatures. In contrast, the negative phonon entropy of vacancy formation decreases the stability of vacancies. The phonon entropy of vacancy formation is about half its configurational counterpart in magnitude, indicating the importance of the phonon entropy of vacancy formation. Previous estimates of the phonon entropy of vacancy formation used a simple model of the phonon spectra of $\mathrm{Fe}$ aluminides $^{39}$ and results from positron annihilation measurements. ${ }^{9}$ With this model, Fähnle et al. ${ }^{11}$ estimated the phonon entropy of vacancy formation on an Fe site of $\Delta S_{\mathrm{ph}}^{\mathrm{V}_{\mathrm{Fe}}}=-0.96 k_{\mathrm{B}}$ / vacancy, consistent with our experiments.

When adding vacancies, the relative importance of $\Delta S_{\mathrm{ph}}^{\mathrm{V}_{\mathrm{Fe}}}$ and $\Delta S_{\mathrm{cf}}^{\mathrm{V}_{\mathrm{Fe}}}$ will change with the vacancy concentration as indicated by Eq. (7). At low vacancy concentrations, $S_{\mathrm{cf}}^{\mathrm{V}_{\mathrm{Fe}}}$ goes as $-2 x_{\mathrm{v}} \ln \left(2 x_{\mathrm{v}}\right)$, whereas $S_{\mathrm{ph}}^{\mathrm{V}_{\mathrm{Fe}}}$ goes as $x_{\mathrm{v}}$. Configurational entropy will therefore dominate in materials with very low vacancy concentrations, but its slow logarithmic singularity allows for the importance of phonon entropy at low vacancy concentrations. For $\mathrm{FeAl}$, at a concentration $x_{\mathrm{v}}=1$ $\times 10^{-7}, \Delta S_{\mathrm{ph}}^{\mathrm{V}_{\mathrm{Fe}}}$ is still approximately $10 \%$ of $\Delta S_{\mathrm{cf}}^{\mathrm{V}_{\mathrm{Fe}}}$. For comparison, these entropies per additional vacancy are approximately equal at the concentration $x_{\mathrm{v}}=0.2$.

The ability of the quasiharmonic model to account for the change in phonon entropy of vacancy formation suggests that volume effects are the primary source of changes in the phonon entropy. In other words, the defects alter phonons by their effect on the specific volume of the crystal. Previously this "volume mechanism" has been used to explain the effects on phonons of order-disorder transitions. ${ }^{40-43}$ From measurements, disordering causes optical phonons to be shifted downward in energy, but their spectra are also broadened. $^{44,45}$ The energy spread of the optical modes is also similar to the effects of vacancies.

Our neutron-scattering samples are very nearly stoichiometric, and the high-pressure samples are slightly Al rich. Based on the work of Kogachi et al., ${ }^{2}$ these samples should have a few triple defects. We expect that monovacancies are predominant, especially for the lower quench temperatures. However, if the triple defect is present, we expect the effect to be somewhat similar to those of the mono-Fe-vacancies because the triple defect also creates a large free volume, and most $1 \mathrm{NN}$ atoms are Al.

Our experimental high-pressure results are in good agree- ment with the ab initio calculations for the bulk modulus, Grüneisen parameter of the Fe modes, and vacancy dependence of the Fe modes. The effects of compression and thermal expansion on the phonon DOS are mutually consistent, and consistent with the volume effect from first principles. This implies that FeAl is approximately quasiharmonic.

\section{CONCLUSIONS}

The effects of vacancies, temperature, and pressure on the phonon entropy and phonon DOS in B2 FeAl were largely due to changes in volume and were generally consistent with the quasiharmonic approximation. Vacancies on the Fe site alter phonons by their effect on the specific volume of the crystal, where the decrease in volume leads to an increase in the phonon energies. The $\mathrm{QH}$ model failed to account for the increase in the number of modes in the acoustic-optic gap with vacancies, which is not predicted by a simple shift in energy associated with changes in the volume. Firstprinciples calculations indicated that these modes are primarily associated with vibrations of $\mathrm{Al}$ atoms in the firstnearest-neighbor shell of the vacancy, with some vibration amplitude also involving the second-nearest-neighbor $\mathrm{Fe}$ atoms.

The phonon entropy of vacancy formation of $-1.7 k_{\mathrm{B}} /$ vacancy is opposite in sign to the corresponding change in configurational entropy and half the magnitude at vacancy concentrations of $1-3 \%$. For dilute isolated Fe vacancies, the relative importance of the configurational entropy per additional vacancy, compared to the vibrational, increases with decreasing concentration $x_{\mathrm{v}}$ as $-2 \ln \left(2 x_{\mathrm{v}}\right)$. Nevertheless, the phonon entropy remains quantitatively significant in $B 2 \mathrm{FeAl}$ to very low concentrations of vacancies.

\section{ACKNOWLEDGMENTS}

We thank C. T. Liu for providing us with the samples for inelastic neutron scattering. This work was benefited from the use of the Intense Pulsed Neutron Source at Argonne National Laboratory. This facility is funded by the U.S. Department of Energy, BES-Materials Science, under Contract No. W-31-109-Eng-38. This work was supported by the Department of Energy through the Basic Energy Sciences Grant No. DE-FG02-03ER46055 and DOE BES-MS Grant No. W-31-109-ENG-38. Portions of this work were performed at HPCAT (Sector 16), Advanced Photon Source (APS), Argonne National Laboratory. The use of the HPCAT facility was supported by DOE-BES, DOE-NNSA (CDAC), NSF, DOD TACOM, and the W.M. Keck Foundation. The use of the APS was supported by DOE-BES under Contract No. DE-AC02-06CH11357.
${ }^{1}$ M. Kogachi and T. Haraguchi, Mater. Sci. Eng., A 230, 124 (1997).

${ }^{2}$ M. Kogachi, T. Haraguchi, and S. M. Kim, Intermetallics 6, 499 (1998).

${ }^{3}$ C. L. Fu, Y.-Y. Ye, M. H. Yoo, and K. M. Ho, Phys. Rev. B 48,
6712 (1993).

${ }^{4}$ C. L. Fu, Phys. Rev. B 52, 3151 (1995).

${ }^{5}$ C. L. Fu and X. Wang, Mater. Sci. Eng., A 239-240, 761 (1997).

${ }^{6}$ J. Mayer, C. Elsässer, and M. Fähnle, Phys. Status Solidi B 191, 283 (1995). 
${ }^{7}$ S. Zaroual, O. Sassi, J. Aride, J. Bernardini, and G. Moya, M. J. Condensed Matter 4, 73 (2001).

${ }^{8}$ G. Bester, B. Meyer, M. Fähnle, and C. L. Fu, Mater. Sci. Eng., A 323, 487 (2002).

${ }^{9}$ R. Würschum, C. Grupp, and H.-E. Schaefer, Phys. Rev. Lett. 75, 97 (1995).

${ }^{10}$ A. Kellou, H. I. Feraoun, T. Grosdidier, C. Coddet, and H. Aourag, Acta Mater. 52, 3263 (2004).

${ }^{11}$ M. Fähnle, G. Bester, and B. Meyer, Scr. Mater. 39, 1071 (1998).

${ }^{12}$ R. O. Simmons and R. W. Balluffi, Phys. Rev. 117, 52 (1960).

${ }^{13}$ M. Kresch, O. Delaire, R. Stevens, J. Y. Y. Lin, and B. Fultz, Phys. Rev. B 75, 104301 (2007).

${ }^{14}$ B. Meyer, V. Schott, and M. Fähnle, Phys. Rev. B 58, R14673 (1998).

${ }^{15}$ G. Grimvall, Thermophysical Properties of Materials (North Holland, Amsterdam, 1999) (enlarged and revised edition).

${ }^{16}$ M. I. Sandakov, V. M. Sandakov, and P. V. Geld, Russ. J. Phys. Chem. 45, 1519 (1971).

${ }^{17}$ T. Gödecke and W. Köster, Z. Metallkd. 75, 161 (1984).

${ }^{18}$ H. J. Leamy, E. D. Gibson, and F. X. Kayser, Acta Metall. 15, 1827 (1967).

${ }^{19}$ H. J. Leamy, Acta Metall. 15, 1839 (1967).

${ }^{20}$ J. Payne and P. D. Desai, Properties of Intermetallic Alloys: I. Aluminides (CINDAS, West Lafayette, IN, 1994).

${ }^{21}$ D. C. Wallace, Statistical Physics of Crystals and Liquids (World Scientific, Singapore, 2002).

${ }^{22}$ E. Sterer, M. Pasternak, and R. Taylor, Rev. Sci. Instrum. 61, 1117 (1990).

${ }^{23}$ R. A. Forman, G. J. Piermarini, J. D. Barnett, and S. Block, Science 176, 284 (1972).

${ }^{24}$ E. E. Alp, W. Sturhahn, T. S. Toellner, J. Zhao, M. Hu, and D. E. Brown, Hyperfine Interact. 144-145, 3 (2002).

${ }^{25}$ M. Seto, Y. Yoda, S. Kikuta, X. W. Zhang, and M. Ando, Phys. Rev. Lett. 74, 3828 (1995).

${ }^{26}$ W. Sturhahn, T. S. Toellner, E. E. Alp, X. Zhang, M. Ando, Y.
Yoda, S. Kikuta, M. Seto, C. W. Kimball, and B. Dabrowski, Phys. Rev. Lett. 74, 3832 (1995).

${ }^{27}$ H. K. Mao, J. Xu, V. V. Struzhkin, J. Shu, R. J. Hemley, W. Sturhahn, M. Y. Hu, E. E. Alp, L. Vocadlo, D. Alfè, G. D. Price, M. J. Gillan, M. Schwoerer-Böhning, D. Häusermann, P. Eng, G. Shen, H. Giefers, R. Lübbers, and G. Wortmann, Science 292, 914 (2001).

${ }^{28}$ W. Sturhahn, Hyperfine Interact. 125, 149 (2000).

${ }^{29}$ G. Kresse and J. Furthmüller, Comput. Mater. Sci. 6, 15 (1996).

${ }^{30}$ G. Kresse and J. Furthmüller, Phys. Rev. B 54, 11169 (1996).

${ }^{31}$ P. E. Blöchl, Phys. Rev. B 50, 17953 (1994).

${ }^{32}$ G. Kresse and D. Joubert, Phys. Rev. B 59, 1758 (1999).

${ }^{33}$ J. P. Perdew, K. Burke, and M. Ernzerhof, Phys. Rev. Lett. 77, 3865 (1996).

${ }^{34}$ F. Birch, Phys. Rev. 71, 809 (1947).

${ }^{35}$ W. Frank, C. Elsässer, and M. Fähnle, Phys. Rev. Lett. 74, 1791 (1995).

${ }^{36}$ G. Kresse, J. Furthmüller, and J. Hafner, EPL 32, 729 (1995).

${ }^{37}$ K. Parlinski, Z-.Q. Li, and Y. Kawazoe, Phys. Rev. Lett. 78, 4063 (1997).

${ }^{38}$ K. Parlinski, Phonon Manual, Version 4.24 (Institute of Nuclear Physics, Cracow, Poland, 2005).

${ }^{39}$ L. Anthony, L. J. Nagel, J. K. Okamoto, and B. Fultz, Phys. Rev. Lett. 73, 3034 (1994).

${ }^{40}$ J. D. Althoff, D. Morgan, D. de Fontaine, M. Asta, S. M. Foiles, and D. D. Johnson, Phys. Rev. B 56, R5705 (1997).

${ }^{41}$ R. Ravelo, J. Aguilar, M. Baskes, J. E. Angelo, B. Fultz, and B. L. Holian, Phys. Rev. B 57, 862 (1998).

${ }^{42}$ A. van de Walle and G. Ceder, Rev. Mod. Phys. 74, 11 (2002).

${ }^{43}$ A. van de Walle, G. Ceder, and U. V. Waghmare, Phys. Rev. Lett. 80, 4911 (1998).

${ }^{44}$ B. Fultz, L. Anthony, L. J. Nagel, R. M. Nicklow, and S. Spooner, Phys. Rev. B 52, 3315 (1995).

${ }^{45}$ B. Fultz, T. A. Stephens, E. E. Alp, M. Y. Hu, J. P. Sutter, T. S. Toellner, and W. Sturhahn, Phys. Rev. B 61, 14517 (2000). 